\title{
Gut Microbiota Alteration Influences Colorectal Cancer Metastasis to the Liver By Remodeling the Liver Immune Microenvironment
}

\section{na yuan}

the first affiliated hospital of Hebei north university https://orcid.org/0000-0001-6268-6106

\section{Xiaoyan Li}

Hebei General Hospital

\section{Meng Wang}

Baoding No1 centeral hospital

\section{Zhilin Zhang}

The First Affiliated Hospital of Hebei North University

\section{Lu Qiao}

Hebei General Hospital

\section{Yamei Gao}

Hebei General Hospital

\section{Xinjian Xu}

the fourth affiliated hospital of Hebei Medical University

Jie Zhi

Hebei General Hospital

Yang Li

affiliated hospital of Hebei University

\section{Zhongxin Li}

The First Affiliated Hospital of Hebei Medical University

Yitao Jia ( jiayitao99@163.com)

the first affiliated hospital of Hebei north university https://orcid.org/0000-0003-2610-9330

\section{Research}

Keywords: colorectal cancer, liver metastasis, gut microbiota, Kupffer cells

Posted Date: April 27th, 2021

DOI: https://doi.org/10.21203/rs.3.rs-429405/v1 
License: (c) (i) This work is licensed under a Creative Commons Attribution 4.0 International License. Read Full License

Version of Record: A version of this preprint was published at Gut and Liver on March 23rd, 2022. See the published version at https://doi.org/10.5009/gnl210177. 


\section{Abstract}

Background: The gut microbiota regulates the liver immune microenvironment in colorectal cancer (CRC) through gut-liver axis. Kupffer cells (KCs) contribute significantly to the formation of pre-metastatic niches (PMNs) for CRC liver metastasis. The aim of our study was to explore the effect of gut microbiota regulating $\mathrm{KCs}$ in $\mathrm{CRC}$ liver metastasis.

Results: Mice treated with different antibiotics were divided into Control group, vancomycin (Vanc) group, colistin (Coli) group, and a group of ampicillin, streptomycin and colistin (ASC). Fewer liver metastases were identified in mice in the ASC group and Coli group than in those in the Control group $(P=0.003, P=$ 0.041 , respectively). An increased proportion of Parabacteroides_goldsteinii, Bacteroides_vulgatus, Bacteroides_thetaiotaomicron, Bacteroides_uniformis in mice of the Coli group were observed in $16 \mathrm{~S}$ rDNA sequencing. Consistent with the Tax4Fun functional prediction, a prominent expansion of hepatic KCs was identified in mice in the ASC and Coli groups compared to those in the Control group $(P=0.001$, $P=0.038$, separately). Meanwhile, Bacteroides_vulgatus was a positive correlation with $\mathrm{KCs}$ contents ( $P$ $=0.011, r=0.705)$. On the other hand, more liver metastases were observed in mice of the Vanc group than those in the Control group $(P=0.028)$. An increased abundance of Parabacteroides_distasonis, Proteus_mirabilis and a remarkable reduction of hepatic KCs were observed in mice in the Vanc group ( $P$ $=0.027)$. Pearson correlation analysis showed Proteus_mirabilis was a negative related to KCs contents $(P=0.028, \mathrm{r}=0.632)$.

Conclusions: An increased abundance of Proteus_mirabilis and a decreased abundance of Bacteroides_vulgatus may be a key factor in CRC liver metastasis, which may be related to the reduction of KCs in the liver.

\section{Introduction}

Colorectal cancer (CRC) is one of the most common malignant tumors worldwide [1], and $15 \sim 25 \%$ of patients with CRC present with liver metastasis at the time of diagnosis [2]. Although advances have been made in the treatment of CRC liver metastasis, over $50 \%$ of patients with CRC occur recurrence and metastasis within 2 years [3]. Thus, it is clinically significant to explore the mechanism underlying CRC liver metastasis to improve the current therapeutic options.

Tumor metastasis is closely related to the tumor microenvironment in situ and in target organs, resulting in significant differences in patient prognosis even the patients with same tumor stage [4]. The liver and gut are incredibly interconnected; indeed, alterations in the CRC microenvironment affect liver homeostasis through gut-liver axis [5]. However, the CRC microenvironment is extremely complex and comprised of gut microbiome and its metabolites or products [6]. Changes in the intestinal flora, in particular, play an important role in the CRC microenvironment, as well as in the occurrence and development of CRC. Increases in Clostridium and decreases in Bacteroides promote CRC liver metastasis [7]. In addition, various bacteria from the gut microbiota may be released into the portal vein 
circulatory system through microbe-associated molecular patterns, thereby reshaping the liver microenvironment [8].

Though the gut microbiome mediates accumulation of hepatic natural killer T (NKT) cells against both primary and metastatic liver tumors, changes in Kupffer cells (KCs), the largest number of residential macrophages, have not been examined [9]. KCs account for approximately $20 \%$ of the non-parenchymal cells in the liver, which play a key role in the process of phagocytic tumors [10]. Meanwhile, KCs regulate $\mathrm{T}$ and NK cell activity and function, KCs activate NK cells to stimulate cytokine secretion, such as granulocyte macrophage colony-stimulating factor and interferon (IFN)- $\gamma$, which in turn enhance the phagocytic activity of KCs [11]. KCs encourage the proliferation of activated CD8 $+\mathrm{T}$ cells in the early period of cancer and promote apoptosis in the late period of cancer [12]. Apart from this, Animal experiments have shown that KCs not only promote T cell apoptosis via the Fas/Fas-L pathway, but also highly express programmed death ligand 1 (PD-L1) to inhibit T cell proliferation and function by directly contacting them $[13,14]$. Thus, the role of KCs in the progression of tumor is extremely complicated.

KCs in hepatic sinusoids contribute to phagocytosis and killing of microbes that originate from the bloodstream; KCs kill green fluorescent protein-expressing B. burgdorferi and perform antigen presentation for NK cells [15]. A higher proportion of lactic acid secreted by bacteria control KCs via reduction of serum lipopolysaccharide (LPS) levels, mitigating the development of non-alcoholic steatohepatitis [16]. LPS is an inflammatory signal that activates Toll-like receptor 4 (TLR4) on KCs, resulting in the inflammation observed in alcoholic liver disease [17]. However, there are limited studies on the effect of the gut microbiota on hepatic KCs in CRC liver metastasis. Therefore, the purpose of this study was to explore the role of the gut microbiota and hepatic KCs in the process of CRC liver metastasis, to provide insights for the development of effective prevention and treatment strategies for CRC liver metastasis.

\section{Materials And Methods}

Cell culture and animal experiments

The mouse colon cancer cell line colon 26 (CT26) (Chinese Academy of Sciences Cell Bank, Shanghai, China) was cultured in Roswell Park Memorial Institute 1640 (Gibco, California, USA) complete medium supplemented with $10 \%$ fetal bovine serum (Gibco, California, USA) and $1 \%$ double antibodies $(100 \mathrm{U} / \mathrm{mL}$ penicillin and $100 \mathrm{\mu g} / \mathrm{mL}$ streptomycin, Gibco, California, USA) at $37{ }^{\circ} \mathrm{C}$ in a $5 \% \mathrm{CO}_{2}$ incubator. Sixty specific-pathogen-free (SPF) male BALB/c mice (aged 6 weeks, Animal Experiment Center of Hebei Medical University, Shijiazhuang, Hebei, China) were divided into four groups, with fifteen mice per group: 1) Control group: received sterile drinking water, 2) Vancomycin (Vanc) group: received $0.25 \mathrm{mg} / \mathrm{mL}$ vancomycin (North China Pharmaceutical Co., Ltd, Shijiazhuang, Hebei, China) alone in sterile drinking water, 3) Colistin (Coli) group: received $2 \mathrm{mg} / \mathrm{mL}$ colistin (Jima Co., Ltd, Shanghai, China)alone in sterile drinking water, and 4) ASC group: received a mix of ampicillin $(1 \mathrm{mg} / \mathrm{mL}$, Lukang Pharmaceutical Co., Ltd, Jining, Shandong, China), streptomycin (5 mg/mL, Meilun Biotechnology Co., Ltd, Dalian, Liaoning, 
China), and colistin $(1 \mathrm{mg} / \mathrm{mL})$ in sterile drinking water [18]. Fecal samples were collected after 2 weeks of antibiotic administration for subsequent $16 \mathrm{~S}$ rDNA sequencing. The antibacterial effect of the antibiotics was evaluated and the model of CRC liver metastasis was established by splenic injection with $1 \times 10^{5}$ CT26 cells as previously described [19] (Fig. 1A).

Bacterial 16S rDNA sequencing

Gene sequencing of $16 \mathrm{~S}$ rDNA was performed using mouse fecal pellets collected before initiation of the CT26 tumor-bearing model. After the antibacterial effect was evaluated, 16S rDNA sequencing was performed. DNA from stool samples was extracted using the cetyltrimethylammonium bromide (Sigmaaldrich, St. Louis, Mo, USA) method. The purity and concentration of DNA was determined using agarose gel electrophoresis. An appropriate amount of DNA was transferred to a centrifuge tube and diluted to 1 $\mathrm{ng} / \mu \mathrm{L}$ with sterile water, and the $\mathrm{V} 3-\mathrm{V} 4$ variable region was amplified using polymerase chain reaction with the primer sequences 515F (5'-GTG CCA GCM GCC GCG GTA A-3') and 806R (5'-GGA CTA CNN GGG TAT CTA AT-3'). The library was constructed using the lon Plus Fragment Library Kit (48 reactions, Thermo Fisher Scientific, Massachusetts, USA). The constructed library was quantified using a Qubit (Thermo Fisher Scientific, Massachusetts, USA) and sequenced using an lon S5TMXL (Thermo Fisher Scientific, Massachusetts, USA). The read sequences were compared to the species annotation database Aby (http:/github.com/torognes/vsearch/), and chimera sequences were examined. Finally, chimera sequences were removed, and only clean reads remained. Uparse software (Uparse v7.0.1001, http://www.drive 5.com/uparse/) was used for clustering under $97 \%$ similarity, while the operational taxonomic units (OTUs) for species classification were obtained after chimera filtering of the clustered sequences. Linear discriminant analysis effect size (LEfSe) software was used to define the filter value as 4. After ensuring that the OTU annotation information originated from the SILVA database (SILVA SSU 138.1, http://www.arb-silva.de/), the whole genome functional information of prokaryotes in the Kyoto Encyclopedia of Genes and Genomes database was annotated using UProC and a protein alignment was performed using a DNA aligner (PAUDA) method corresponding to the SILVA database, thus realizing the functional prediction of Tax4Fun. Immunohistochemistry

Mouse liver tissues were harvested, embedded in paraffin blocks, and cut into $4 \mu \mathrm{m}$ sections. After dewaxing, hydrating, antigen repair, and incubation in $3 \% \mathrm{H}_{2} \mathrm{O}_{2}$ solution for $15 \mathrm{~min}$, monoclonal rabbit anti-mouse F4/80 primary antibody (1:500, Servicebio, Wuhan, Hubei, China) was added. Color was developed using a 3, 3'-diaminobenzidine staining solution immunohistochemistry kit (Zsbio, Peking, China). Following microscopic (Nikon, Tokyo, Japan) observation, three randomly selected high-power fields were used to acquire images, and the average optical density of each protein was calculated using ImageJ software (Version 1.8.0, National Institutes of Health, New York, USA).

\section{Statistical analysis}

SPSS 19.0 software (SPSS Inc., Chicago, IL, USA) was used for statistical analysis. Data are expressed as the mean \pm the standard deviation. After normal distribution and variance homogeneity testing, one-way 
analysis of variance and the least significant difference methods were used for multiple comparisons. Pearson correlation analysis was used to analyze the correlation of $\mathrm{KCs}$ and differential bacteria between the Control group and Coli group, the Control group and Vanc group. The differences of gut microbiota between groups were analyzed statistically using the R software. Student's $t$-test and Wilcoxon rank-sum were used for comparison between two groups, while Tukey's post hoc test and a Wilcoxon test were performed using the agricolae-package for comparison of more than two groups. $P<0.05$ was considered significantly different.

\section{Results}

Alterations in the gut microbiota influenced CRC liver metastasis

Spontaneous CRC liver metastasis was induced using an intrasplenic tumor injection in BALB/c mice, as previously described [9] (Fig. 1A). ASC antibiotics were added to sterile drinking water to deplete the gut commensal bacteria [20] (Fig. 1B). Fewer liver metastases were identified in mice in the ASC group ( $P=$ 0.003). Colistin alone treatment (Coli group) inhibited the liver metastases of CRC $(P=0.041)$. Whereas, vancomycin alone treatment (Vanc group) promoted the liver metastases of CRC $(P=0.028)$. Mice treated with vancomycin targeting gram-positive bacteria had more liver metastases than that of mice treated with colistin targeting gram-negative bacteria $(P<0.01)$; compared to the Vanc group, a robust decrease of liver metastasis was observed in mice in the ASC group $(P<0.01)$. Similar results were observed for liver volume, a smaller liver volume was observed in mice of the ASC group, whereas, a larger liver volume was performed in mice of the Vanc group; meanwhile, the liver volume in mice of the Vanc group was larger than that of mice in the Coli group and ASC group $(P<0.05)$ (Fig. 1B-C).

To better understand the role of the gut microbiome in response to CRC liver metastasis, fecal samples were prospectively collected and the landscape of the gut microbiome was assessed in all available samples via $16 \mathrm{~S}$ rDNA sequencing, including samples from the Control group, Vanc group, Coli group and ASC group (6 fecal samples per each group). Since ASC treatment efficiently reduced gut commensal bacterial load, it resulted in the complete elimination of gut bacteria. A Venn graph revealed that a total of 395 (Control group), 258 (Vanc group), and 373 (Coli group) species were identified, and a total of 220 species were common among the groups (Fig. 1D). Principal coordinate analysis (PCA) demonstrated that the microbial community structure was clearly separated between the Vanc and Coli groups, while that in the Coli and Control groups was not completely separated (Fig. 1E). Moreover, significant differences were observed in the Alpha diversity of the gut microbiota between the Coli and Vanc groups using several methods such as Chao1, Shannon, and Simpson indexes; while Coli group significantly increased the diversity of the microbiota with Shannon and Simpson diversity (Fig. 1F). From Beta diversity analysis, relative to the Control group, the difference coefficient of gut microbiome diversity in the Coli and Vanc groups was 0.145 and 0.509 , respectively. Furthermore, a correlation coefficient of 0.466 in commensal bacteria discrepancy was presented between the Coli and Vanc groups (Fig. 1G). The above results suggested that modulating gut commensal bacteria affected CRC liver metastasis; community richness and diversity increased could depress the liver metastasis of CRC. 
Specific gut microbiome is responsible for liver metastasis of CRC

It was determined if differences existed in the gut microbiota among three groups, high-dimensional class comparisons of common bacterial taxa were revealed through LEfSe Bar and Cladogram. At the phylum level, Firmicutes and Bacteroidetes were rich in the Coli group; Proteobacteria was rich in the Vanc group. At the species level, Bacteroides_vulgatus, Bacteroides_uniformis and Helicobacter_mastomyrinus were rich in the Coli group, while Proteus_mirabilis was rich in the Vanc group (Fig. 2A-B). Compared to the Control group based on a Wilcoxon rank-sum test, it was performed that an increased abundance of Parabacteroides_goldsteinii, Bacteroides_vulgatus, Bacteroides_thetaiotaomicron, Bacteroides_uniformis were enriched in the Coli group, while an increased proportion of Parabacteroides_distasonis, Proteus_mirabilis were enriched in the Vanc group at the species level $(P<0.05$, Fig. 2C-D). These results suggested the increasing of Parabacteroides_goldsteinii, Bacteroides_vulgatus, Bacteroides_thetaiotaomicron, Bacteroides_uniformis might inhibit the CRC liver metastasis, the increasing of Parabacteroides_distasonis, Proteus_mirabilis could promote liver metastasis of the CRC.

Function prediction of the differential bacteria among three groups

Whether the underlying mechanism from commensal bacteria was responsible for the observed liver metastasis from the function prediction of Tax4Fun, it was observed that the differential gene functions in the three groups mainly included gene, cell motility, various metabolisms, immune system, and transport (Fig. 3A). Further pair-wise comparisons were performed to uncover the distinction of the immune system, which showed that there were obvious differences in immune system between the Coli and Control groups, as well as both the Vanc and Control groups, the differential functions among three groups contained bacterial motility protein, bacterial secretion system, two-component system, additionally, NOD-like receptor signaling pathway and IL-17 signaling pathway (Fig. 3B-D). The Tax4Fun function prediction specified that gut microbiome alteration affected CRC liver metastasis, which was correlated with IL-17 signaling pathway.

The role of hepatic KCs in liver metastasis of CRC and its correlationship with differential microbiota.

According to Tax4Fun function prediction above, immune signals, in particular, IL-17 signaling pathway was remarkably different in the three groups. To explore the mechanism behind tumor suppression, the number of hepatic KCs in CT26 tumor-bearing mice was examined by immunohistochemistry. A prominent expansion of hepatic KCs were observed in mice in the ASC group and the Coli group ( $P=$ $0.001, P=0.038$, respectively). While a remarkable reducing of hepatic KCs was observed in mice in the Vanc group $(P=0.027)$. Similar to the liver metastasis and liver volume results, accumulation of hepatic KCs was also observed in mice in the ASC and Coli groups compared to that of mice in the Vanc group ( $P$ $<0.01, P<0.01$, respectively) (Fig. 4A). These findings suggested that depressing gram-negative bacteria promoted KCs leading to an anti-tumor metastatic function, while depleting gram-positive bacteria reduced KCs resulting in a stronger pro-tumor metastatic function. 
To define the exact role of gut microbiota regulating hepatic KCs accumulation, the relationship between differential bacteria of the three groups and KCs contents were tested. There was positive correlation between Bacteroides_vulgatus and KCs contents $(P=0.011, r=0.705)$; while there was negative correlation between Proteus_mirabilis and KCs contents $(P=0.028, \mathrm{r}=0.632)$ (Fig. 4B-C).

Taken together, an increased abundance of Bacteroides_vulgatus and a decreased amount of Proteus_mirabilis might be involved in the regulation of hepatic KCs number, resulting in the different tendency of CRC liver metastasis.

\section{Discussion}

The precise mechanism underlying CRC liver metastasis has not been elucidated. Recently, the concept of the gut-liver axis has provided the basis for studying the relationship between the liver and intestinal diseases; gut microbiota enter the liver through the portal vein system, which may affect the liver microenvironment and participate in CRC liver metastasis [17]. Here, models of CRC liver metastasis were established using mice administered different antibiotics; it was observed that the mice in the vancomycin treatment group had more liver metastases, whereas those in the colistin treatment group and mixed treatment group had less liver metastases. There was an increased abundance of Proteus_mirabilis in the Vanc group; in contrast, Bacteroides_vulgatus was enriched in the Coli group prior to metastasis. The effect of gut microbiota compositional diversities on CRC liver metastasis maybe related to KCs in the liver.

CRC liver metastasis models were established in mice with different gut microbiota. The number of liver metastases between mice in the Vanc group was more than those in the Control group. However, a previous study has reported a significantly reduced number of liver metastases in mice in the Vanc group compared to those in the Control group [9]. These conflicting results may be associated with different feeding doses of antibiotics, the duration time of vancomycin treatment, number of tumor cells inoculated via the spleen, selection of animal strains or animal feeding conditions. In addition, fewer liver metastases were observed in mice in the ASC group than those in the Control group, indicating that depletion of intestinal symbiotic bacteria inhibited CRC liver metastasis. Similar results are reported by Sethi et al. who established liver metastasis models of pancreatic cancer, colon cancer, or melanoma in mice using antibiotics to exhaust gut flora; in all models, a broad-spectrum cocktail of antibiotic treatment (vancomycin, neomycin, metronidazole, ampicillin, and amphotericin B) significantly reduces the occurrence of liver metastasis [21]. Remarkably, there were more liver metastases observed in mice in the Control group than those in the Coli group. In a hapten-induced rat model of colitis, enteral administration of colistin significantly reduces the fecal count of gram-negative bacilli and attenuates the damage of systemic endotoxemia in colitis [22]. In addition, colistin may be a valuable treatment for clinically severe forms of Escherichia coli (E.coli) 0157:H7, as intragastric administration of E.coli results in larger CRC tumors and more liver metastatic nodes in CRC models [23, 24]. 
According to the PCA, Alpha, and Beta diversity analyses of the gut microbiota, a distinct separation in bacterial community composition was observed among mice in three groups. To better identify the specific commensal bacteria responsible for CRC liver metastasis, differences in commensal bacteria were analyzed between mice in the Vanc and those in the Control group, as well as both of the Coli group and Control group. Increased abundance of Parabacteroides_distasonis, Proteus_mirabilis could accelerate CRC liver metastasis, while increased abundance of Parabacteroides_goldsteinii, Bacteroides_vulgatus, Bacteroides_thetaiotaomicron, Bacteroides_uniformis might inhibit CRC liver metastasis. Similarly, bacteria belonging to the genus Bacterioides, for example, Bacteroides_vulgatus, have anti-tumor effects, which may be associated with the immune response mediated by their activation of myeloid differentiation protein-2/TLR4 and the TLR2 signaling pathway [25, 26]. In the cancer patients, T cell responses specific for Bacteroides_thetaiotaomicron or Bacteroides_fragilis were associated with the efficacy of CTLA-4 blockade [27].

Just as the Tax4Fun prediction, the underlying functional difference among mice in the three groups was clarified to be associated with immune signaling. Specially, IL-17 is a tumor-promoting cytokine that critically regulates inflammatory responses in KCs in an experimental model of alcohol-induced hepatocellular carcinoma [28]. Cellular components involved in PMN formation in CRC liver metastasis consist of various cells, including non-resident cells such as bone marrow-derived cells and resident cells such as hepatic stellate cells, KCs, and liver sinusoidal endothelial cells (LSECs), among which, KCs play a key role in CRC liver metastasis [29]. In the current study, consistent with the results of liver metastasis nodes, an increasing abundance of KCs in mice in the ASC group compared to that of mice in the Control group suggested gut commensal bacteria exhaustion likely improved KC accumulation. Importantly, lower KC abundance was observed in mice in the Vanc group, while more KC abundance was performed in mice in the Coli group. It is reported that vancomycin induced elevated level of IL-25, in vitro and in vivo, IL-25 induced alternative activation of macrophages (M1 and M2-subtype alternation) promoted the growth of hepatocellular carcinoma [30]. A study showed that colistin treatment could increase the phagocytic ability of macrophage via p38/mitogen-activated protein kinase pathway [31]. In addition, patients with partial hepatectomy are prone to intrahepatic recurrence, partly due to a reduction in the number of liver-resident KCs, which induce the activation of TNF-a, receptor-interacting protein kinase 3 , and caspase-1 to recruit other monocyte-derived cells that are beneficial to tumor growth [32].

In our study, a prominent expansion of hepatic KCs were observed in mice in the Coli group, while a remarkable reduction of hepatic KCs was observed in mice in the Vanc group compared to the Control group, suggesting that KCs accumulation might inhibit liver metastasis of CRC. In addition, the raising of Bacteroides_vulgatus and the decreasing of Proteus_mirabilis were positively correlated with the number of hepatic KCs, suggesting the majority of Bacteroides_vulgatus may be a key bacterium leading to KCs accumulation. After injection with a combination of T. hyodysenteriae and Bacteroides_vulgatus in mice, which marked macrophage aggregation and neutrophil necrosis were examined by light and electron microscopy [33]. The microbiota stimulates inflammation, in particular, Proteus_mirabilis induces IL-1 $\beta$ release upon intestinal injury, which was largely mediated by intestinal Ly6C (high) monocytes [34]. However, our current study had limitations; only one cell line (CT26) was used, other hepatic immune cells 
were not detected, and the KC subtype was not identified. Additionally, the specific mechanism involved requires further study.

In conclusion, the alteration of gut microbiota diversity exerted an influence on CRC liver metastasis. An increased abundance of Bacteroides_vulgatus and a decreased abundance of Proteus_mirabilis may be a key factor in CRC liver metastasis, which may be related to KCs accumulation in the liver.

\section{Abbreviations}

CRC: colorectal cancer; Vanc:vancomycin; Coli:colistin; ASC:a group of ampicillin, streptomycin and colistin; NKT:natural killer T; KCs:Kupffer cells; IFN-ץ:interferon (IFN)-ү; LPS:lipopolysaccharide.

\section{Declarations}

\section{Authors' contributions}

All authors participated in the operation of experiment; Yitao Jia and Zhongxin Li conceived and designed the study; Na Yuan, Xiaoyan Li, Meng Wang, Lu Qiao, Yamei Gao performed the mice experiments and $\mathrm{H}$ \& E; Xinjian Xu and Jie Zhi collected the materials and datas; Yang Li and Zhilin Zhang analyzed the data; $\mathrm{Na}$ Yuan drafted and wrote the manuscript; All authors read and approved the final manuscript.

\section{Author details}

${ }^{1}$ Department of Oncology, Hebei Medical University, No. 361 Zhongshan East Road, Changan District, 050017, Shijiazhuang, Hebei, China. ${ }^{2}$ The Third Department of Oncology, Hebei General Hospital, No. 348 Heping West Road, Xinhua District, 050051, Shijiazhuang, Hebei, China. ${ }^{3}$ Department of Radiotherapy, The First Affiliated Hospital of Heibei North University, No.12 Changqing Road, Qiaoxi District, Zhangjiakou, 075000, Hebei, China. ${ }^{4}$ Department of Clinical Psychology, Baoding No.1 Central Hospital, No.320 Changcheng North Avenue, Baoding, 071000, Hebei, China. ${ }^{5}$ Department of Thoracic Surgery, The Fourth Hospital of Hebei Medical University, No.12 Jiankang Road, 050011, Shijiazhuang, Hebei, China. ${ }^{6}$ Department of Oncology, Affiliated Hospital of Hebei University, No.212 Yuhua East Road, Baoding, 071000 , Hebei, China. ${ }^{7}$ Department of General Surgery, The First Hospital of Hebei Medical University, No.89 Donggang Road, Yuhua District, 050030, Shijiazhuang, Hebei, China.

\section{Acknowledgements}

The authors wish to express their sincere thanks to all those who have lent their hands in the course of writing this paper.

\section{Conflict of interest}

The authors declare that they have no conflict of interest. 
Availability of data and materials

All data generated or analyzed during this study are included in this published article.

\section{Consent for publication}

Not applicable

\section{Funding}

This work was supported by The Specialist Capacity Building and Leader Development Program Funded from Hebei government in 2018 (361003).

\section{Ethical Approval}

This study was approved by the Administration Committee of Experimental Animals, Heibei Medical University, Hebei Province, China.

\section{Statement of Human and Animal Rights}

All of the experimental animal procedures were conducted in accordance with the requirements of the Regulations of Hebei Medical University on the Management and Use of Experimental Animals (License: SYXK (Hebei) 2015-0065).

\section{References}

1. Kashyap D, Sharma A, Tuli HS, Sak K, Mukherjee T, et al. Molecular targets of celastrol in cancer: Recent trends and advancements. Crit Rev Oncol Hematol. 2018;128:70-81.

2. Lim CJ, Lee YH, Pan L, Lai L, Chua C, et al. Multidimensional analyses reveal distinct immune microenvironment in hepatitis B virus-related hepatocellular carcinoma. Gut,2019,68(5):916-927.

3. Imai K, Allard MA, Benitez CC, Vibert E, Sa Cunha A, et al. Early Recurrence After Hepatectomy for Colorectal Liver Metastases: What Optimal Definition and What Predictive Factors. Oncologist,2016,21(7):887-94.

4. Jia B. Commentary. Gut microbiome-mediated bile acid metabolism regulates liver cancer via NKT cells. Front Immunol. 2019;10:282.

5. Golonka RM, Vijay-Kumar M. Atypical immunometabolism and metabolic reprogramming in liver cancer: Deciphering the role of gut microbiome. Adv Cancer Res. 2021;149:171-255.

6. Cruz B, Sarandy MM, Messias AC, Gonçalves RV, Ferreira C, et al. Preclinical and clinical relevance of probiotics and synbiotics in colorectal carcinogenesis: a systematic review. Nutr Rev. 2020;78(8):667-87.

7. Li Y, Wang S, Sun Y, Xu W, Zheng H, et al. Apple polysaccharide protects ICR mice against colitis associated colorectal cancer through the regulation of microbial dysbiosis. Carbohydr Polym. 
2020;230:115726.

8. Clarke TB, Davis KM, Lysenko ES, Zhou AY, Yu Y, et al. Recognition of peptidoglycan from the microbiota by Nod1 enhances systemic innate immunity. Nat Med. 2010;16(2):228-31.

9. Ma C, Han M, Heinrich B, Fu Q, Zhang Q, et al. Gut microbiome-mediated bile acid metabolism regulates liver cancer via NKT cells. Science,2018,360(6391).

10. Huang H, Lu Y, Zhou T, Gu G, Xia Q. Innate Immune Cells in Immune Tolerance After Liver Transplantation. Front Immunol. 2018;9:2401.

11. Kuniyasu Y, Marfani SM, Inayat IB, Sheikh SZ, Mehal WZ. Kupffer cells required for high affinity peptide-induced deletion, not retention, of activated CD8 + T cells by mouse liver. Hepatology. 2004;39(4):1017-27.

12. Tu Z, Bozorgzadeh A, Pierce RH, Kurtis J, Crispe IN, et al. TLR-dependent cross talk between human Kupffer cells and NK cells. J Exp Med. 2008;205(1):233-44.

13. Chen Y, Liu Z, Liang S, Luan X, Long F, et al. Role of Kupffer cells in the induction of tolerance of orthotopic liver transplantation in rats. Liver Transpl. 2008;14(6):823-36.

14. Gong J, Cao D, Chen Y, Li J, Gong J, et al. Role of programmed death ligand 1 and Kupffer cell in immune regulation after orthotopic liver transplantation in rats. Int Immunopharmacol. 2017;48:816.

15. Lee WY, Moriarty TJ, Wong $\mathrm{CH}$, Zhou $\mathrm{H}$, Strieter RM, et al. An intravascular immune response to Borrelia burgdorferi involves Kupffer cells and iNKT cells. Nat Immunol. 2010;11(4):295-302.

16. Okubo H, Kushiyama A, Sakoda $H$, Nakatsu Y, lizuka $M$, et al. Involvement of resistin-like molecule $\beta$ in the development of methionine-choline deficient diet-induced non-alcoholic steatohepatitis in mice. Sci Rep. 2016;6:20157.

17. Ishida K, Kaji K, Sato S, Ogawa H, Takagi H, et al. Sulforaphane ameliorates ethanol plus carbon tetrachloride-induced liver fibrosis in mice through the Nrf2-mediated antioxidant response and acetaldehyde metabolization with inhibition of the LPS/TLR4 signaling pathway. J Nutr Biochem. 2021;89:108573.

18. Lee JG, Lee YR, Lee AR, Park CH, Han DS, et al. Role of the global gut microbial community in the development of colitis-associated cancer in a murine model. Biomed Pharmacother. 2021;135:111206.

19. Eggert T, Wolter K, Ji J, Ma C, Yevsa T, et al. Distinct Functions of Senescence-Associated Immune Responses in Liver Tumor Surveillance and Tumor Progression. Cancer Cell,2016,30(4):533-547.

20. Xu X, Lv J, Guo F, Li J, Jia Y, et al. Gut Microbiome Influences the Efficacy of PD-1 Antibody Immunotherapy on MSS-Type Colorectal Cancer via Metabolic Pathway. Front Microbiol. 2020;11:814.

21. Sethi V, Kurtom S, Tarique M, Lavania S, Malchiodi Z, et al Gut Microbiota Promotes Tumor Growth in Mice by Modulating Immune Response. Gastroenterology,2018,155(1):33-37.e6. 
22. Gardiner KR, Erwin PJ, Anderson NH, McCaigue MD, Halliday MI, et al. Lactulose as an antiendotoxin in experimental colitis. Br J Surg. 1995;82(4):469-72.

23. Percivalle E, Monzillo V, Pauletto A, Marone P, Imberti R. Colistin inhibits E. coli 0157:H7 Shiga-like toxin release, binds endotoxins and protects Vero cells. New Microbiol. 2016;39(2):119-23.

24. Li R, Zhou R, Wang H, Li W, Pan M, et al. Gut microbiota-stimulated cathepsin K secretion mediates TLR4-dependent M2 macrophage polarization and promotes tumor metastasis in colorectal cancer. Cell Death Differ. 2019;26(11):2447-63.

25. Uronis JM, Mühlbauer M, Herfarth HH, Rubinas TC, Jones GS, et al. Modulation of the intestinal microbiota alters colitis-associated colorectal cancer susceptibility. PLoS One,2009,4(6):e6026.

26. Di Lorenzo F, Pither MD, Martufi M, Scarinci I, Guzmán-Caldentey J, et al. Pairing Bacteroides vulgatus LPS Structure with Its Immunomodulatory Effects on Human Cellular Models. ACS Cent Sci,2020,6(9):1602-1616.

27. Vétizou M, Pitt JM, Daillère R, Lepage P, Waldschmitt N, et al. Anticancer immunotherapy by CTLA-4 blockade relies on the gut microbiota. Science,2015,350(6264):1079-84.

28. Ma HY, Yamamoto G, Xu J, Liu X, Karin D, et al. IL-17 signaling in steatotic hepatocytes and macrophages promotes hepatocellular carcinoma in alcohol-related liver disease. J Hepatol. 2020;72(5):946-59.

29. Keirsse J, Van Damme H, Geeraerts X, Beschin A, Raes G, et al. The role of hepatic macrophages in liver metastasis. Cell Immunol. 2018;330:202-15.

30. Li Q, Ma L, Shen S, Guo Y, Cao Q, et al. Intestinal dysbacteriosis-induced IL-25 promotes development of HCC via alternative activation of macrophages in tumor microenvironment. J Exp Clin Cancer Res. 2019;38(1):303.

31. Wang J, Shao W, Niu H, Yang T, Wang Y, et al. Immunomodulatory Effects of Colistin on Macrophages in Rats by Activating the p38/MAPK Pathway. Front Pharmacol. 2019;10:729.

32. Hastir JF, Delbauve S, Larbanoix L, Germanova D, Goyvaerts C, et al. Hepatocarcinoma Induces a Tumor Necrosis Factor-Dependent Kupffer Cell Death Pathway That Favors Its Proliferation Upon Partial Hepatectomy. Front Oncol. 2020;10:547013.

33. Albassam MA, Olander HJ, Thacker HL, Turek JJ. Electron microscopic studies on the interaction between normal mice peritoneal phagocytes and Treponema hyodysenteriae, Treponema innocens and Bacteroides vulgatus. Can J Vet Res. 1986;50(1):88-95.

34. Seo SU, Kamada N, Muñoz-Planillo R, Kim YG, Kim D, et al. Distinct Commensals Induce Interleukin$1 \beta$ via NLRP3 Inflammasome in Inflammatory Monocytes to Promote Intestinal Inflammation in Response to Injury. Immunity,2015,42(4):744-55.

\section{Figures}


A

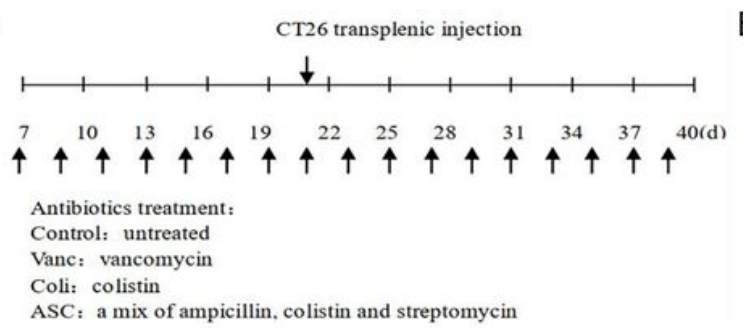

B

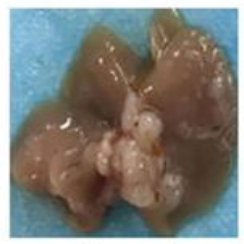

Control

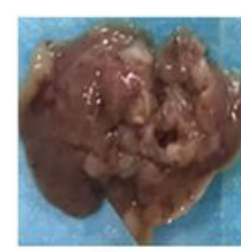

Vanc

D
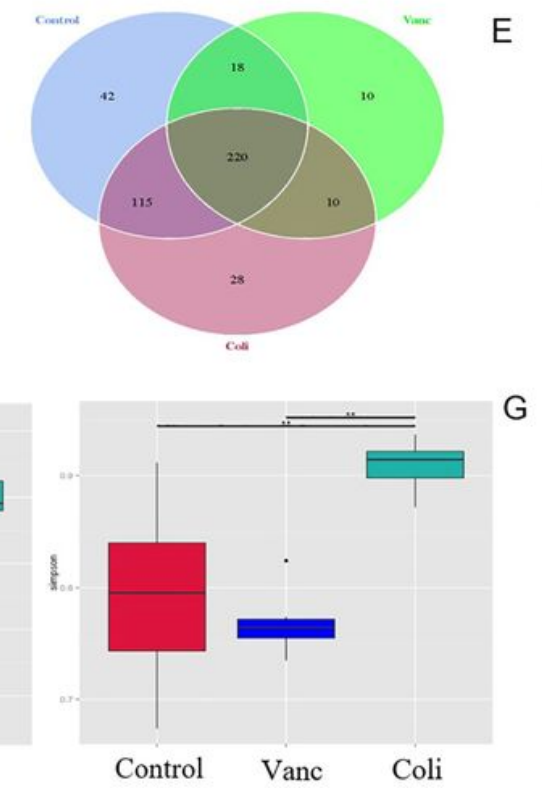

Control

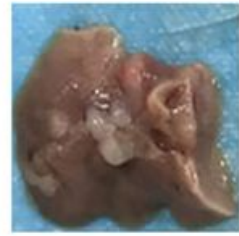

Coli

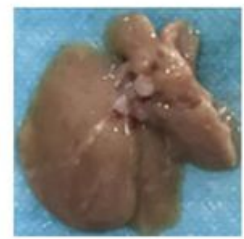

ASC
C
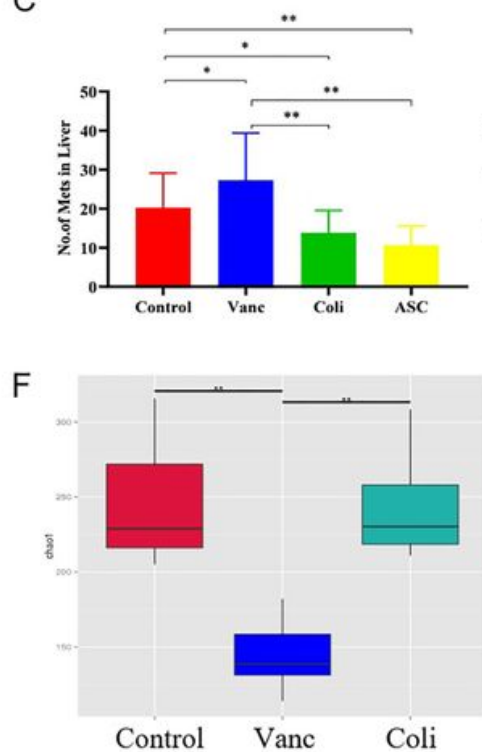
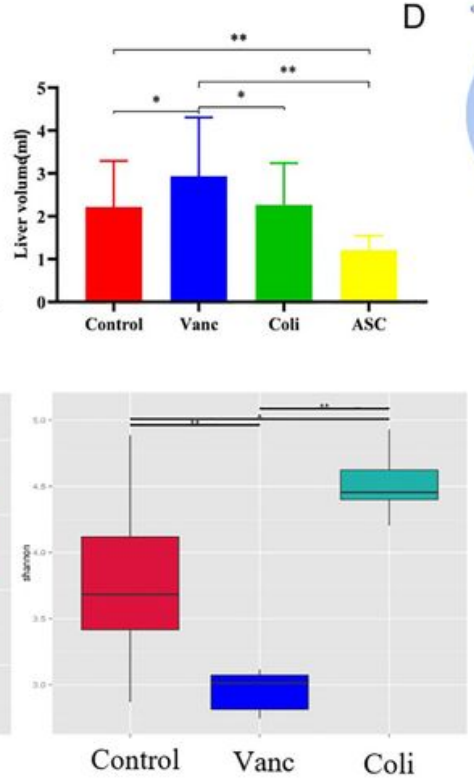

E

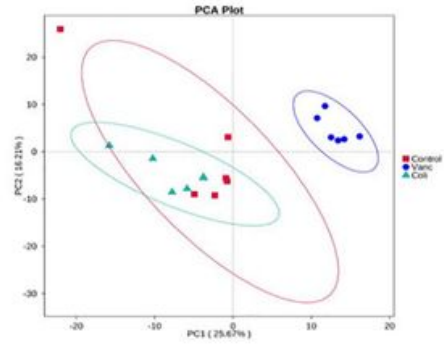

G

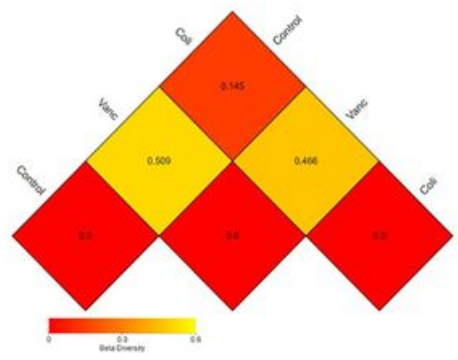

Figure 1

Changes in gut microbiota and colorectal cancer (CRC) liver metastasis induced by different antibiotics. A Schematic diagram of the mouse experimental process of CRC liver metastasis under the action of different antibiotics (Upper arrow: CRC liver metastasis model, as established by CT26 transplenic injection; Lower arrow: antibiotic treatment). B Image of CRC liver metastases treated with different antibiotics at the end of the experiment. C Number of CRC liver metastases and liver volume in different groups ( $n=15 /$ group) (a mouse in the Vanc group and a mouse in the ASC group were lost during the experiment from emaciation and intestinal obstruction). D Venn diagram of the total number of species was among the Control, Vanc, and Coli groups ( $n=6 /$ group). E Principal coordinates analysis (PCA) analysis of operational taxonomic units (OTUs) in the Control, Vanc, and Coli groups. F Alpha diversity analysis of the gut microbiome was among the Control, Vanc, and Coli groups. Alpha diversity includes the Shannon, Chao1 and Simpson indexes. G Beta diversity was reflected by the weighted Unifrac distance. 
A
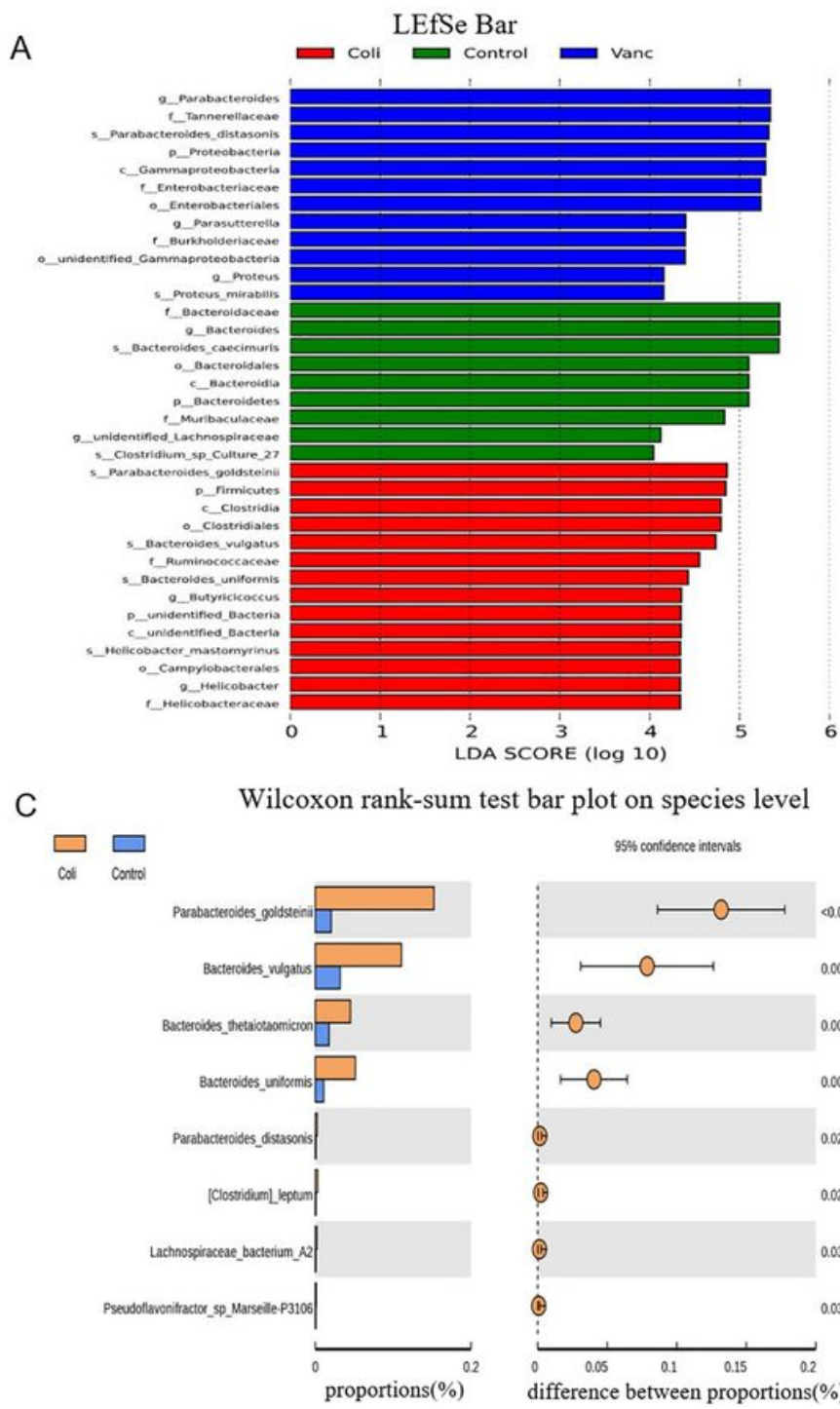

B

Cladogram

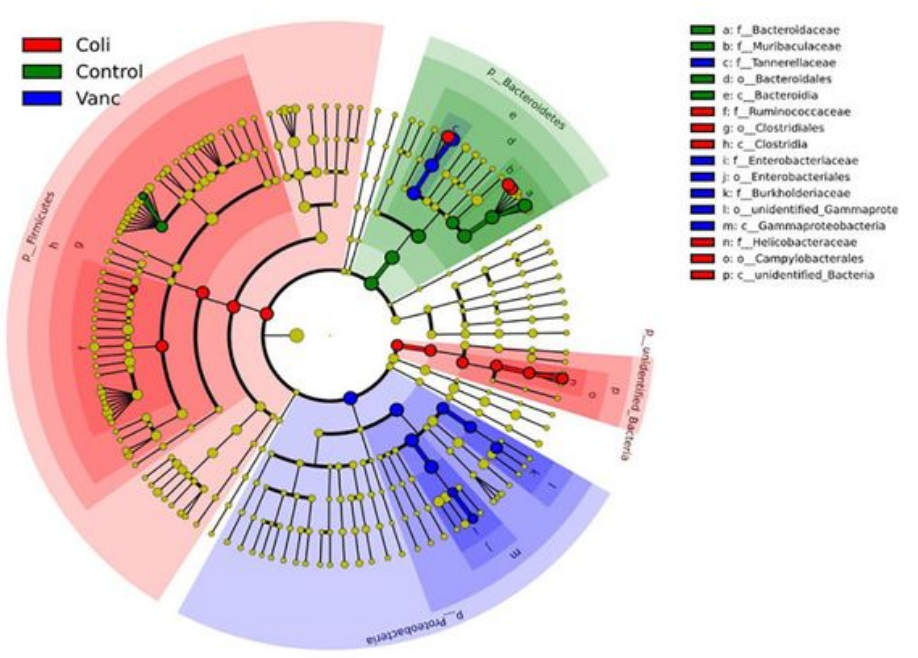

D

Wilcoxon rank-sum test bar plot on species level

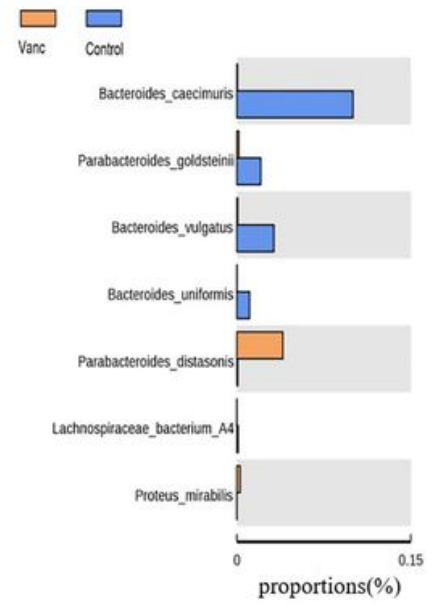

$95 \%$ confidence intervals

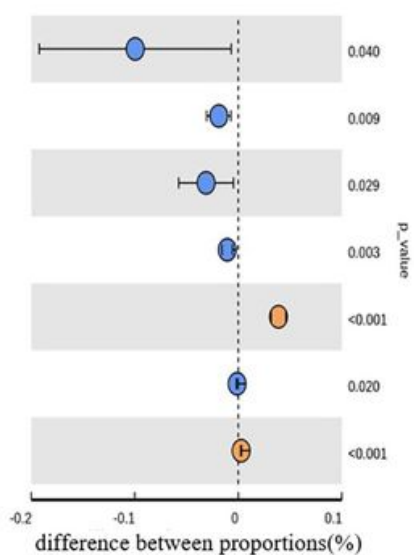

Figure 2

The comparison of gut microbiota composition was performed among three groups. A Linear discriminant analysis effect size (LEfSe) for differentially abundant taxa from the three groups. $P=0.05$ using the Wilcoxon rank-sum test, linear discriminant analysis (LDA) score > 4. B Taxonomic cladogram from the LEfSe showing differences in fecal taxa. Dot size is proportional to the abundance of the taxon ( $n=6 /$ group). C Compositional differences at the species level in the gut microbiome between the Control and Coli groups. D Compositional differences at the species level in the gut microbiome between the Control and Vanc groups. 
A

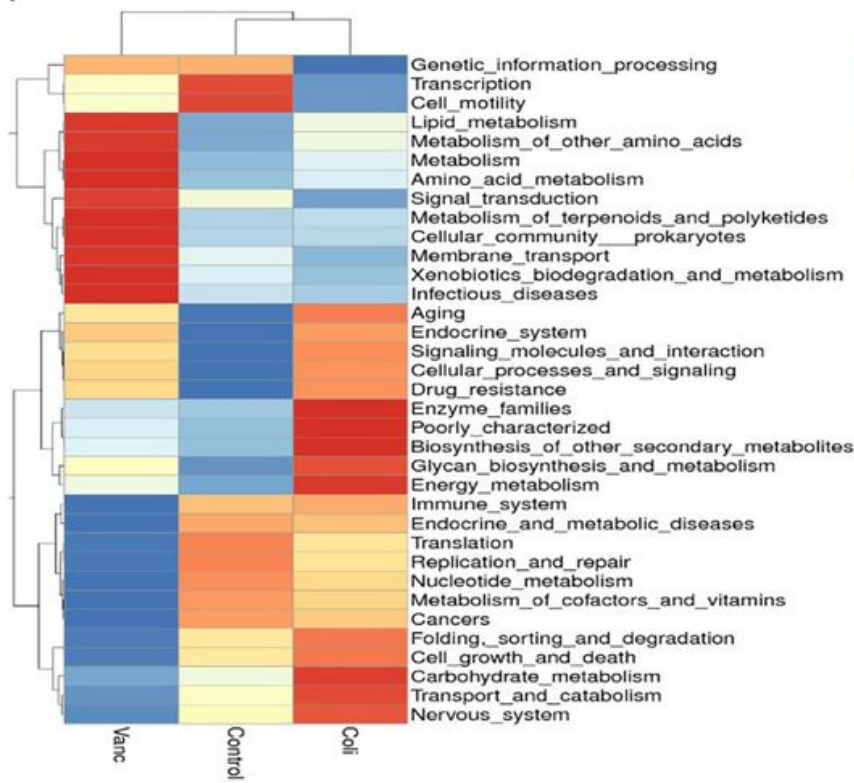

C Immune signal between Vanc group and Control group

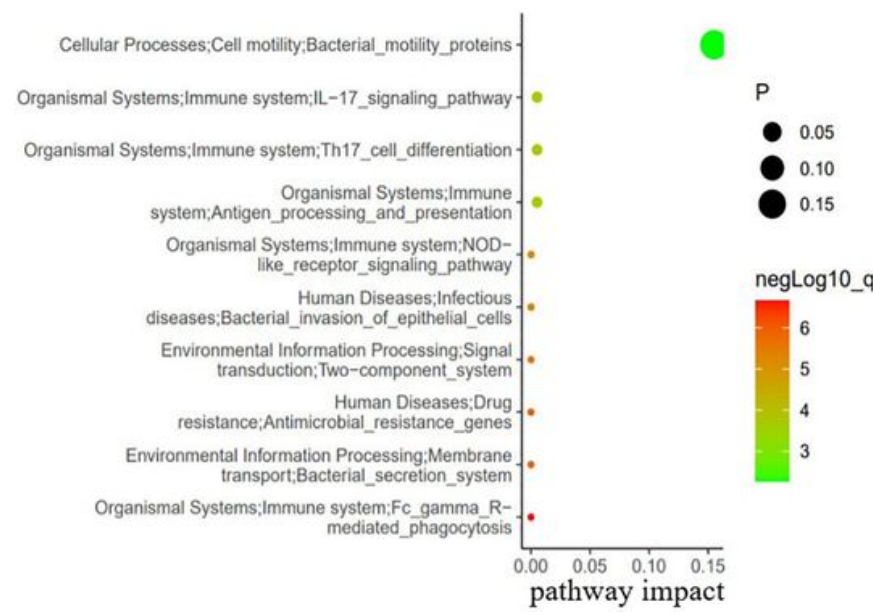

Immune signal between Coli group and Control group
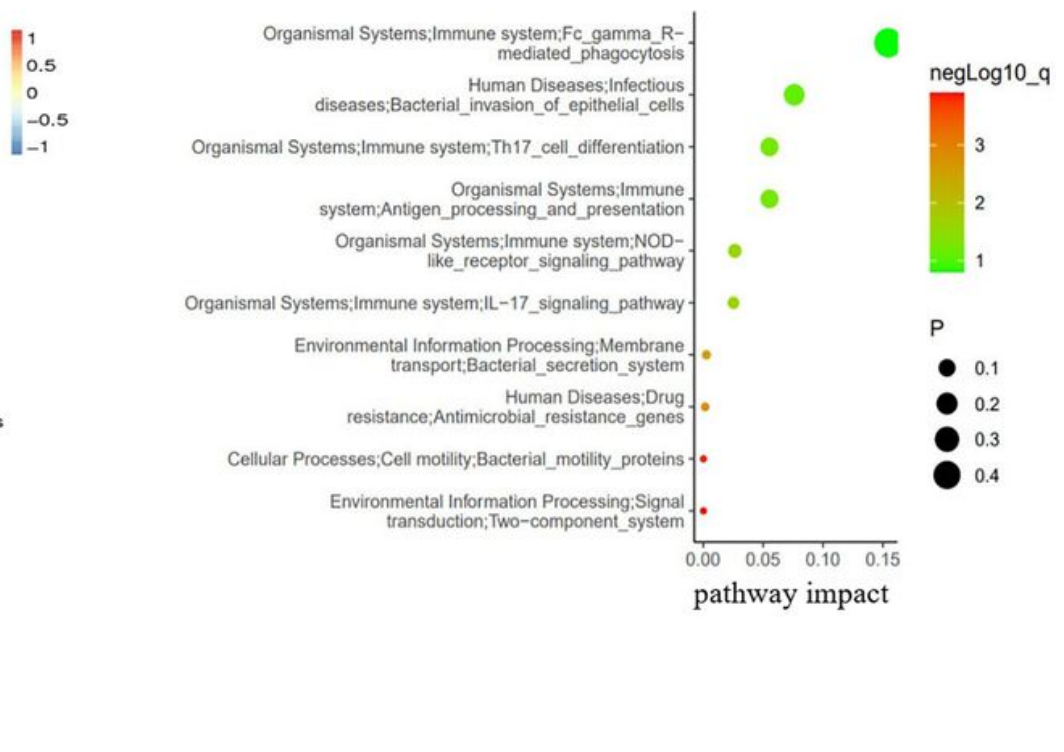

D Immune signal between Vanc group and Coli group

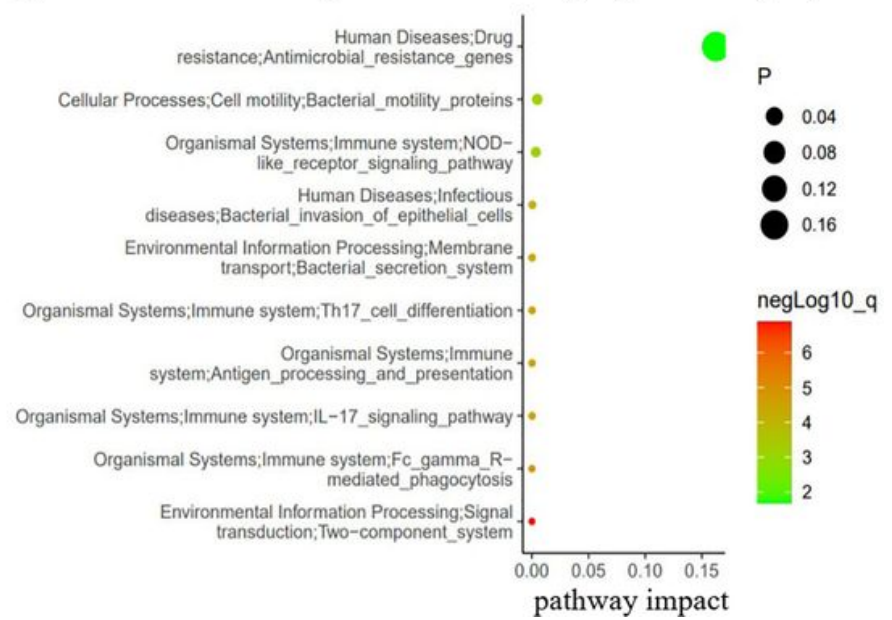

Figure 3

Tax4Fun functional prediction analysis for differential bacteria among the three groups. A Tax4Fun functional prediction in the Control, Vanc, and Coli groups was showed in Heatmap. B Pathway enrichments of immune system, environmental information and cellular processes were compared in the Control and Coli groups (dot size symbolizes the P value). C Pathway enrichments of immune system, environmental information and cellular processes were compared in the Control and Vanc groups. D Pathway enrichments of immune system, environmental information and cellular processes were compared in the Coli and Vanc groups. 
A

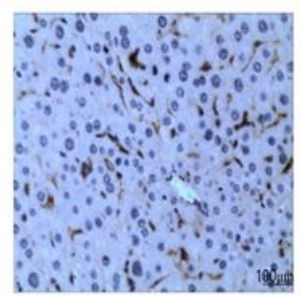

Control

C
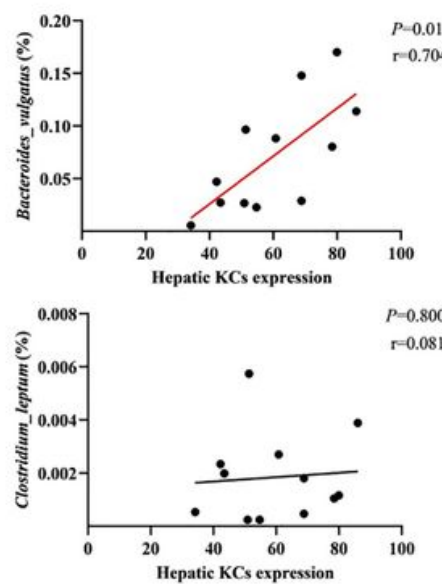

$\mathrm{D}$
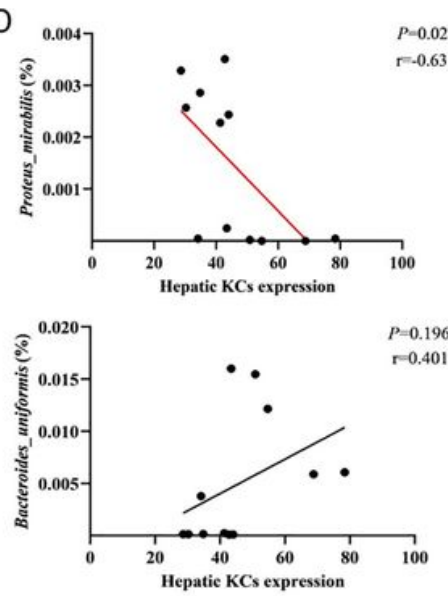

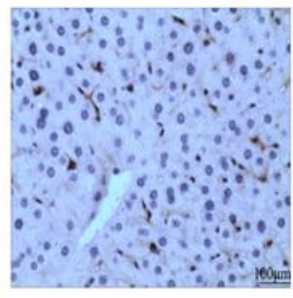

Vanc

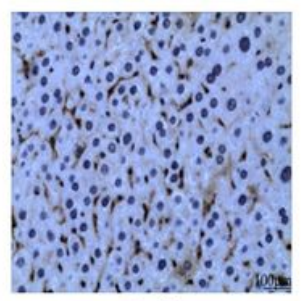

Coli

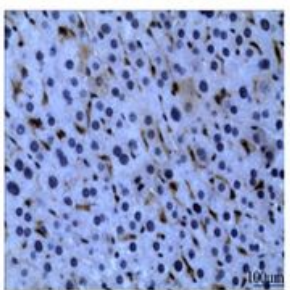

ASC

$B$
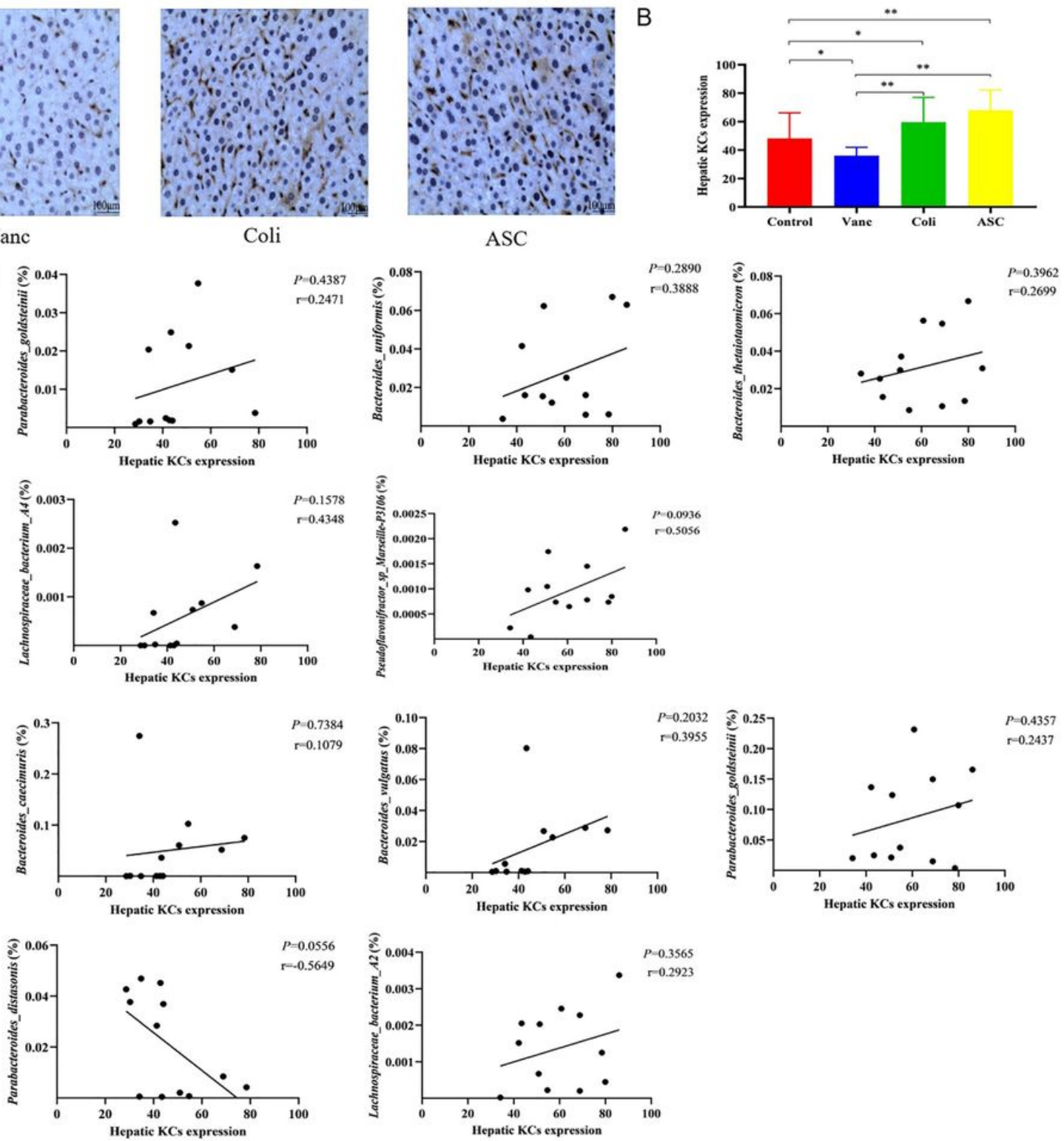

Figure 4

Correlationship of the differential microbiota and hepatic Kupffer cell $(\mathrm{KC})$ accumulation, tumor inhibition was exhibited in three groups. A Hematoxylin and eosin (H \& E) staining images and quantification of hepatic KCs from different gut flora backgrounds ( $n=5 /$ group) (200x); B Analysis of correlation with KCs and the species level of differential microbiota between the Control group and the Coli group. C Analysis of correlation with KCs and the species level of differential microbiota between the Control group and the Vanc group. 